\title{
Design of a Single-and Double -Row Planetary Gear Oil-electric Hybrid Power Automobile Transmission System
}

\author{
Gong Wenzi \\ Wuxi Vocational Institute of Commerce, Wuxi, Jiangsu 214153, China
}

\begin{abstract}
Keywords: Oil-electric Hybrid Power Automobile; Single and double-row planetary gear type; Design of transmission system; Energy recovery
\end{abstract}

\begin{abstract}
This paper introduces the transmission system of a single, double-row planetary gear oil-electric hybrid power automobile. The main drive motor and auxiliary drive motor cooperate with the corresponding planetary gear mechanism, which can realize different working modes and timely startup of gasoline engine. When the charging condition is available, external 220V AC power supply can charge the power battery.
\end{abstract}

\section{Introduction}

Hybrid power automobile refers to such a type of cars carrying different sources of power, which can run simultaneously or respectively with different power sources according to the driving needs of vehicles; the driving modes include series, parallel and mixed types. At present, the most common oil-electric hybrid power automobiles are powered by gasoline engines and motors. Hybrid power automobiles can automatically run in the pure electric driving mode, engine independent driving mode or hybrid driving mode according to driving conditions, and have the function of energy recovery when necessary. Although oil-electric hybrid power automobile can't achieve zero emissions, it can effectively solve the problems such as short driving range and incomplete charging infrastructure, and the function of energy recovery and external AC charging interface can further improve its efficiency and reduce emissions pollution; therefore, the oil-electric hybrid power automobile has better environmental protection, energy saving and efficiency compared with traditional internal combustion engine vehicles, and will have good development trends in the present period and in the near future.

\section{System Composition}

As shown in figure 1, the oil-electric hybrid power automobile transmission system shown in figure 1 , is composed of engine, two motors, two planetary lines, four brakes, two clutches and driving wheels. The engine is a petrol engine. The first planetary line is a two-stage planetary gear mechanism, and the second planetary line is a single-stage planetary gear mechanism. Motor 1 and motor 2 are both permanent magnet brushless DC motor; Motor 2 as the main driving motor, can also be used as a generator when the energy is recovered or start the engine in time to work together with motor 1 . Motor 1 is generator and auxiliary drive motor, and can also be independent or start the engine in time to work together with motor 2. The clutches can connect or disconnect two components as needed. The brake can be used to fix a component according to the need.

\section{Control Principle}

The block diagram of control principle is shown in figure 2, of which the engine is controlled by the engine control unit, the clutches and brakes are controlled by a hybrid power control unit, motor 1 and motor 2 are controlled by frequency converter, and the motor control unit and hybrid power control unit communicate through CAN bus.

\section{Working Process}

The ignition switch is closed and the hybrid power automobile enters the working state. When the 
gear switch is neutral, the engine, motor 1 and motor 2 do not work, all the clutches are in a separate state and all the brakes do not work. After the gear switch is put into gear, the hybrid power control unit determines the working conditions of the clutches and brakes, and whether motor 1, motor 2 and the engine work and the working mode by analysis and calculation according to the signals of accelerator pedal, gear, brake, speed and other sensors. The working state, working mode and switching of each actuator are automatically controlled, which also determines the working status of the hybrid power automobile. The "startup conditions", "low speed and small load condition", "medium high speed and heavy load conditions", "sharp deceleration, braking and overspeeding conditions while driving forward", "engine overhaul or debugging status", and the working process of "AC charging" are introduced below respectively.

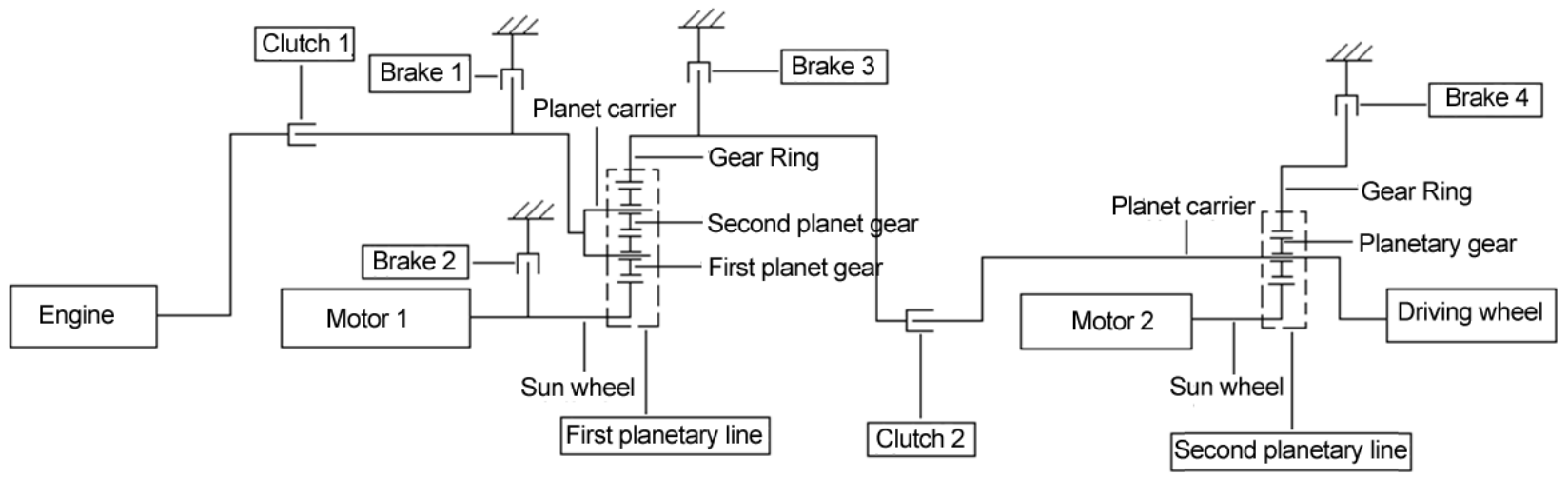

Figure 1-Composition of transmission system

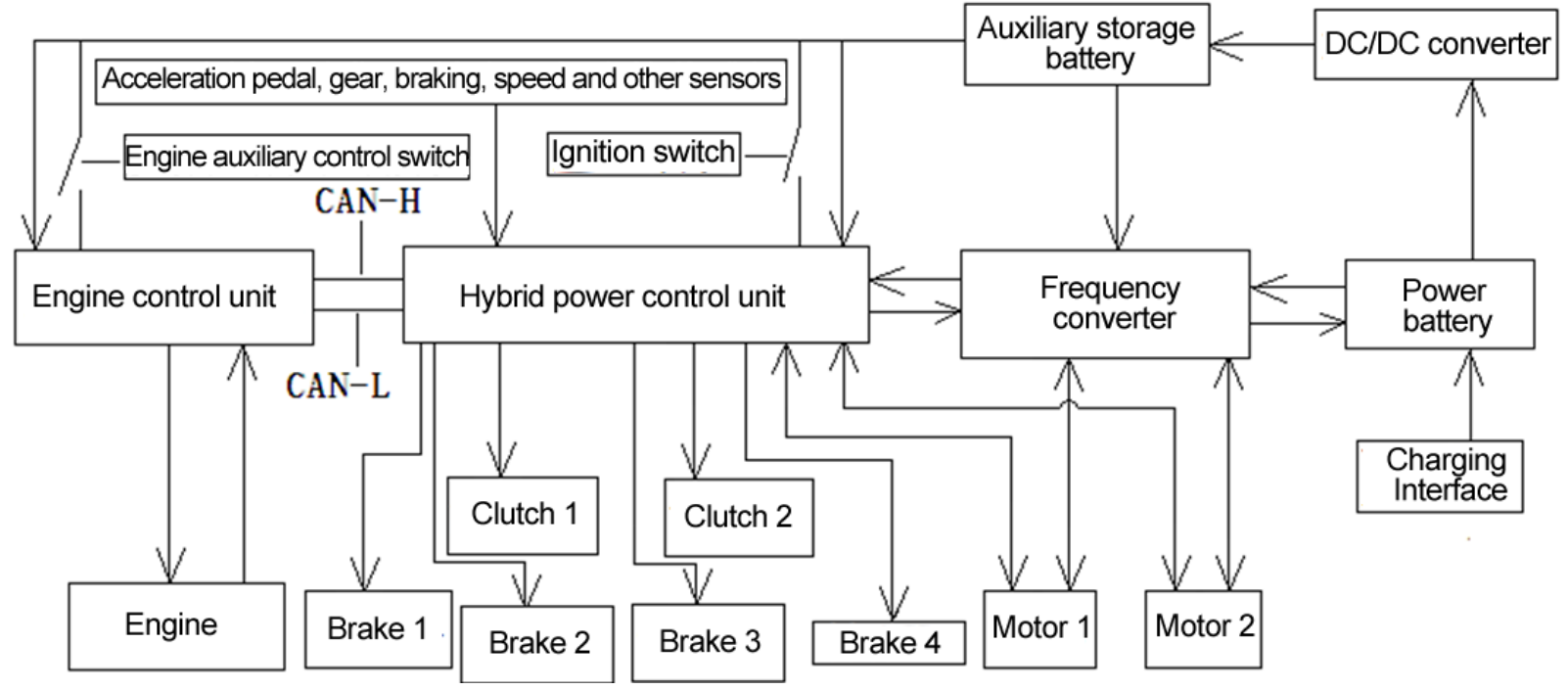

Figure 2- Control schematic diagram

\section{Startup Conditions}

In the pure electric drive mode, the engine and motor 1 don't work, clutch 1 and clutch 2 are in a separate state, and brake 1, brake 2 and brake 3 don't work, and the brake 4 brakes the gear of the 2n planetary line. Put into the forward gear, and the motor 2 is turning to drive the car forward, and when reversing, the motor 2 reverses to drive the car to start and back off.

\section{Low Speed and Small Load Condition}

Sufficient Power Battery. In the pure electric driving mode, the engine does not work, and there are two modes that is, independent driving of motor 2 and joint driving of motor 1 and motor 2; the specific mode is determined by the hybrid power control unit according to the need. The independent driving mode of motor 2 is the same as the starting condition. In the joint driving mode of motor 1 and motor 2, clutch 1 is in a separate state, clutch 2 is closed, brake 1 and brake 4 are in 
the braking state, but brake 2 and brake 3 don't work. Moving forward or backward is determined by the motor turning or reversing. Motor 1 and motor 2 turning forward when moving forward, while the motor turns in the opposite direction when reversing.

Insufficient Power Battery. Forward Driving. Clutch 1 and clutch 2 are in the closed state, and clutch 4 is in the braking state; brake 1, brake 2 and brake 3 don't work, and both motor 1 and motor 2 work timely to start the engine. After the engine has started, brake 2 turns into the working state, the engine drives wheels to run, but motor 1 does not work, and motor 2 turns into the generator while the engine's spare energy is charged to the power battery through motor 2 .

Reverse Driving. If the power battery is under voltage not seriously, the driving mode is the same as the "low-speed and small-load condition" when the "power battery is sufficient". If the battery power is under voltage seriously, the instrument will show the battery serious under voltage; at this point, clutch 1 is closed and clutch 2 is in a separate state, brake 1 brake 22 and brake 4 don't work and brake 3 is in the braking state, and motor 1 reverses to start the engine; After the engine has started, the working state of motor 1 converts to the power generation mode to charge the power battery, but motor 2 does not work. When the power battery reaches a certain capacity, the car runs into the reversing state, and at the time the driving mode is the same as "low speed and small load condition" when the "power battery is sufficient".

\section{Medium High Speed and Heavy Load Condition}

This condition is only used for driving forward. Clutch 1 and clutch 2 are in the closed state, and clutch 4 is in the braking state; brake 1, brake 2 and brake 3 don't work, and both motor 1 and motor 2 work timely to start the engine. After the engine has started, brake 2 turns into the working state, but motor 1 does not work, the engine drives wheels to run, and motor 2 turns into the generator while the engine's spare energy is charged to the power battery through motor 2 .

\section{Sharp Deceleration, Braking and over Speeding Conditions when Driving Forward}

When the car is urgently decelerating or braking while driving forward, or when the speed is more than the set top speed, the working mode of the system will automatically convert to the mode of energy recovery; at this point, the engine does not work, both clutch 1 and clutch 2 are in a separate state, brake 4 is in the braking state, brake 1, brake 2 and brake 3 don't work, motor 1 doesn't work, and motor 2 changes to the generation condition; the vehicle kinetic energy turns into electrical energy to charge the power battery, for energy recovery.

\section{Engine Overhaul or Debug Status}

When the engine needs to be overhauled or debugged, the gear switch should be put to neutral. (1) In case that it does not need to start the engine; the auxiliary engine control switch of the engine is on the ignition position, the engine control unit enters into the working state, and the engine can be tested with multimeter and other relevant instruments; at this point, the ignition switch does not need to be closed. (2) In case that there is a need to start the engine, when the ignition switch is closed, and the auxiliary control switch of the engine is on the startup position, the hybrid control unit controls the clutch 1 to be closed, the clutch 2 to be separated, and the brake 3 under the braking state; the brake 1, brake 2 and brake 4 don't work, the motor 1 reverses, and the engine starts running. After the engine has started, the auxiliary control switch of the engine should return to the ignition position. When the clutches are all separated and the brakes do not work, the engine can be overhauled or debugged.

\section{AC Charging}

When the charging condition is available, an external 220V AC power supply can charge the power battery. 


\section{Conclusion}

The system has the function of energy recovery, which can reduce fuel consumption and brake heat, improve the economy of automobiles, and extend the service life of the braking system. It has AC charging function, and can be connected with $220 \mathrm{~V}$ AC power supply to charge the power battery. The engine control unit and the hybrid power control unit can cooperate and coordinate with each other to realize optimal working mode.

\section{References}

[1] Jiang Kejun, He Ren, Liang Chi, Wang Shan. Review of hybrid power automobile coupling technology [J]. Mechanical transmission,2015,39 (04):175-181.

[2] Zhao Zhenning. Construction, principle and maintenance of hybrid power automobile [M] Beijing: Beijing Institute of Technology Press, 2015.

[3] Tan Kecheng, Wan Dong. Construction, principle and maintenance of hybrid power automobile [M] Beijing: Chemical Industry Press, 2016.

[4] Chen Shehui. Construction and maintenance of hybrid power automobile [M] Beijing: China Labor \& Social Security Publishing House, 2013.

[5] Li Wei. Construction, principle and troubleshooting of new energy vehicles [M] Beijing: Chemical Industry Press, 2015.

[6] Zhang Jinzhu. Structure, principle and maintenance of hybrid power automobile [M] Beijing: Chemical Industry Press, 2011.

[7] Wu Xingmin, Zhang Bo, Wang Yanguang. Construction, principle and maintenance of electric vehicle [M] Beijing: Beijing Institute of Technology Press, 2015. 